\title{
On ovals on Riemann surfaces
}

\section{Grzegorz Gromadzki}

Dedicated to the memory of my father

\begin{abstract}
We prove that $k(k \geq 9)$ non-conjugate symmetries of a Riemann surface of genus $g$ have at most $2 g-2+2^{r-3}(9-k)$ ovals in total, where $r$ is the smallest positive integer for which $k \leq 2^{r-1}$. Furthermore we prove that for arbitrary $k \geq 9$ this bound is sharp for infinitely many values of $g$.
\end{abstract}

\section{Introduction.}

Let $X$ be a compact Riemann surface of genus $g \geq 2$. By a symmetry of $X$ we mean, in this paper, an antiholomorphic involution $\sigma$ which has fixed points. A surface admitting a symmetry is said to be symmetric. The principal motivation for the study of symmetric Riemann surfaces comes from the theory of algebraic curves. A compact Riemann surface $X$ corresponds to a smooth complex projective algebraic curve and symmetries, non-conjugate in the group $\operatorname{Aut}^{ \pm}(X)$ of all automorphisms of $X$, give rise to non-isomorphic over the reals, real models of the curve. A classical theorem of Harnack [8] states that the set $F(\sigma)$ of fixed points of $\sigma$ consists of $\|\sigma\|$ in range $1 \leq\|\sigma\| \leq g+1$ disjoint simple closed curves to which, following Hilbert's terminology, we shall refer to as the ovals of $\sigma$. The number of ovals of a symmetry equals the number of connected components of the corresponding real model.

In this paper we are looking for the maximal number $\omega(g, k)$ of ovals that $k$ non-conjugate symmetries of a Riemann surface $X$ of genus $g$ may admit. This question was investigated at the end of seventies by $\mathrm{S}$. 
M. Natanzon in [11], [12] and [13] who proved many results concerning low values of $k$. In particular, he proved that $\omega(g, k) \leq 2 g+2^{k-1}$ for $2 \leq k \leq 4$ and that this bound is attained respectively for every $g$ congruent to 1 modulo $2^{k-2}$. However the problem of finding the bound for $\omega(g, k)$ for $k \geq 5$ has not been solved up to now. Results concerning surfaces of even $g$, which by [6] have at most 4 non-conjugate symmetries with fixed points, have been recently obtained in [7].

Recently this question was taken up by Singerman [17] who showed that for arbitrary $k$ there exist infinitely many values of $g$ for which there exists a Riemann surface of genus $g$ having $k$ non-conjugate symmetries and $M_{k}=2 g+2^{k-3}(9-k)-2$ ovals in total and he conjectured that this is the best bound. From the recent paper of Natanzon [14] it follows that this indeed is the case in the special situation of separable symmetries. Observe that for $k=3$ and 4 the Singerman and Natanzon bounds coincide without this additional assumption.

Here we show that for $k \geq 9, \omega(g, k) \leq 2 g-2+2^{r-3}(9-k)$, where $r$ is the smallest positive integer for which $k \leq 2^{r-1}$. Furthermore we prove that for arbitrary $k \geq 9$ this bound is sharp for infinitely many values of $g$. In particular there are no $k>9$ for which Singerman's conjecture is true. It is true for $k=9$ and probably true for $5 \leq k \leq 8$.

\section{Preliminaries.}

The results announced in the previous section will be proved using combinatorial techniques based on Fuchsian and NEC groups. The basic results concerning these matter can be found in [3]. However for the reader's convenience we point out some of the most important concepts and results.

The starting point in a combinatorial study of compact Riemann surfaces of genus $g \geq 2$ is the Riemann uniformization theorem by which each such surface can be represented as the orbit space of the hyperbolic plane $\mathcal{H}$ under the action of some Fuchsian surface group $\Gamma$. Furthermore having a surface $X$ so represented its group of automorphisms can be represented as $\Delta / \Gamma$ for another Fuchsian group $\Delta$. Now the orbit space of $X$ under the action of some symmetry $\sigma$ has a structure of Klein surface and the point is that the counterpart of these results for Klein surfaces also holds (see [10] and [15]), where NEC groups play the role of Fuchsian groups.

The algebraic structure of an NEC group $\Lambda$ is determined by its 
signature $([9],[18])$ which is a symbol of the form

$$
\left(g^{\prime} ; \pm ;\left[m_{1}, \ldots, m_{r}\right] ;\left\{C_{1}, \ldots, C_{k}\right\}\right),
$$

where the numbers $m_{i} \geq 2$ are called the proper periods, $C_{i}$ are the $s_{i \text {-uples }}\left(n_{i 1}, \ldots, n_{i s_{i}}\right)$ called the period cycles, the numbers $n_{i j} \geq 2$ are the link periods and $g^{\prime} \geq 0$ is said to be the orbit genus of $\Lambda$. A surface $N E C$ group is an NEC group with only empty period cycles and without proper periods, i.e, an NEC group with signature $\left(g^{\prime} ; \pm ;[-],\{(-), . k .,(-)\}\right)$, a Fuchsian group can be regarded as an $N E C$ group with signature $\left(g^{\prime} ;+;\left[m_{1}, \ldots, m_{r}\right] ;\{-\}\right)$ and finally a Fuchsian surface group is a Fuchsian group with signature $\left(g^{\prime} ;+;[-] ;\{-\}\right)$. A group $\Lambda$ with signature (1) has a presentation with canonical generators

$$
x_{i}, \quad 1 \leq i \leq r, \quad e_{i}, c_{i j}, \quad 1 \leq i \leq k, 0 \leq j \leq s_{i},
$$

and

$$
a_{i}, b_{i} \text { or } d_{i}, \quad 1 \leq i \leq g^{\prime},
$$

and relators

$$
x_{i}^{m_{i}}, \quad 1 \leq i \leq r, \quad c_{i j}^{2},\left(c_{i j-1} c_{i j}\right)^{n_{i j}}, c_{i 0} e_{i}^{-1} c_{i s_{i}} e_{i},
$$

with $1 \leq i \leq k, 0 \leq j \leq s_{i}$, and

$$
x_{1} \cdots x_{r} e_{1} \cdots e_{k} a_{1} b_{1} a_{1}^{-1} b_{1}^{-1} \cdots a_{g^{\prime}} b_{g^{\prime}} a_{g^{\prime}}^{-1} b_{g^{\prime}}^{-1},
$$

or

$$
x_{1} \cdots x_{r} e_{1} \cdots e_{k} d_{1}^{2} \cdots d_{g^{\prime}}^{2},
$$

according as the sign is + or - .

Finally the hyperbolic area of an arbitrary fundamental region of an NEC group $\Lambda$ with signature (1) equals

(2) $\mu(\Lambda)=2 \pi\left(\varepsilon g^{\prime}-2+k+\sum_{i=1}^{r}\left(1-\frac{1}{m_{i}}\right)+\frac{1}{2} \sum_{i=1}^{k} \sum_{j=1}^{s_{i}}\left(1-\frac{1}{n_{i j}}\right)\right)$,

where $\varepsilon=2$ if there is a " + " sign and $\varepsilon=1$ otherwise. If $\Gamma$ is a subgroup of finite index in $\Lambda$, then it is an NEC group itself and we have the Hurwitz-Riemann formula

$$
[\Lambda: \Gamma]=\frac{\mu(\Gamma)}{\mu(\Lambda)} .
$$




\section{Centralizers, conjugacy classes and some combinatorics.}

A group $G$ is said to be abstractly orientable if it admits an epimorphism $\alpha: G \longrightarrow \mathbb{Z}_{2}=\{ \pm 1\}$ which will be called an abstract orientation of $G$. An element $g$ of $G$ is said to be orientation preserving (respectively orientation reversing) subject to the orientation $\alpha$ if $\alpha(g)=+1$ (respectively $\alpha(g)=-1$ ). Examples of orientable groups are provided by proper NEC groups and groups $\operatorname{Aut}^{ \pm}(X)$ of all automorphisms of symmetric Riemann surfaces $X$. The first lemma of this section is an immediate consequence of Sylow theorems.

Lemma 3.1. Let $2^{n}$ be the biggest power of 2 that divides the order of an abstractly oriented finite group $G$. Then $G$ has at most $2^{n-1}$ conjugacy classes of orientation reversing elements of order 2 .

Proof. Indeed let $S$ be a Sylow subgroup of $G$. Then each conjugacy class has a representative in $S$. So the lemma follows since $\operatorname{Ker} \alpha_{\mid S}$, which consists of orientation preserving elements is a subgroup of $S$ of index 2 .

Lemma 3.2. Let $G$ be a finite group and let $y_{1}, y_{2}$ be two elements of order 2 whose product has order $n$. Then the order of the centralizer $\mathrm{C}\left(G, y_{i}\right)$ of $y_{i}$ in $G$ does not exceed $2|G| / n$ for $i=1,2$.

Proof. Let $H$ be the group generated by $y_{1}$ and $y_{2}$ and observe first that $\mathrm{C}\left(H, y_{i}\right)=\mathbb{Z}_{2}$ or $\mathbb{Z}_{2} \oplus \mathbb{Z}_{2}$ according as $n$ is odd or even. Fix a system $X$ of representatives for the cosets of $G / H$. Then each element $g$ of $G$ can be represented as $g=y x$ for some $y \in H$ and $x \in X$ uniquely determined. Now assume that both $g=y x$ and $g^{\prime}=y^{\prime} x \in \mathrm{C}\left(G, y_{i}\right)$. Then $H \ni y^{\prime} y^{-1}=g^{\prime} g^{-1} \in \mathrm{C}\left(G, y_{i}\right)$. Thus $y^{\prime} y^{-1} \in \mathrm{C}\left(H, y_{i}\right)$ and so the lemma follows.

Finally in this section we prove the following elementary combinatorial lemma that we shall need in the sequel.

Lemma 3.3. Assume that $k, k \geq 3$ labels are used to label $s$ points situated on a circle in such a way that no two consecutive points have the same label. Then at least $k-1$ points have neighbours with distinct labels. 
Proof. We shall prove the lemma by induction on $s$. Observe first that $s \geq k$ and that the cases $s=3$ and $s=4$ are trivial. So assume that $s \geq 5$. There is nothing to prove if no point has neighbours with the same label; here $s$ points have neighbours with distinct labels. So assume that there are three consecutive points $i-1, i, i+1$, say with labels $1, k$ and 1 respectively and consider the induced configuration of $s-2$ points $1, \ldots, i-1, i+2, \ldots, s$.

Assume first that some of these points have label $k$. Then by the inductive hypothesis $t \geq k-1$ points have neighbours with distinct labels. If, in the new configuration, the point $i-1$ has neighbours with the same label then in the former configuration these $t$ points have neighbours with distinct labels whilst if $i-1$ has neighbours with distinct labels then in the former configuration $t-1$ of these points and one among $i-1$ and $i+1$ has neighbours with distinct labels.

If none of the points $1, \ldots, i-1, i+2, \ldots, s$ has label $k$ then we have a configuration of $s-2$ points on circle labeled by $k-1$ labels. For $k=3, s$ is even and we see that $i-1$ and $i+1$ have neighbours with distinct labels. So assume that $k>3$. Then by the inductive hypothesis, $k-2$ of these points have distinct labels. So the assertion follows since in this case these points and $i+1$ have neighbours with distinct labels in the former configuration.

\section{Symmetries of Riemann surfaces and their ovals.}

Let $\mathrm{Aut}^{+}(X)$ be the group of orientation preserving automorphisms of a compact Riemann surface $X$ represented as $\mathcal{H} / \Gamma$. Then $\operatorname{Aut}^{+}(X)=\Delta / \Gamma$ for some Fuchsian group $\Delta$ which is the normalizer of $\Gamma$ in $\operatorname{PSL}(2, \mathbb{R})$. Now, $X$ is symmetric if and only if there exists an $N E C$ group $\Lambda$ containing $\Delta$ as a subgroup of index 2 and $\Gamma$ as a normal subgroup. In such case $G=\Lambda / \Gamma=\operatorname{Aut}^{ \pm}(X)$ is the group of all automorphisms of $X$, including those that reverse its orientation. Let $\theta: \Lambda \longrightarrow G$ be the canonical projection. A symmetry of $X$ is an element $\sigma \in \operatorname{Aut}^{ \pm}(X) \backslash \operatorname{Aut}^{+}(X)$ of order 2. Let us denote by $\langle\sigma\rangle$ the group generated by $\sigma$ and represent it as $\Gamma_{\sigma} / \Gamma$ for some $N E C$ subgroup $\Gamma_{\sigma}$ of $\Lambda$. Then the orbit space $X /\langle\sigma\rangle \cong \mathcal{H} / \Gamma_{\sigma}$ is a Klein surface whose boundary coincides with $\operatorname{Fix}(\sigma)$. So $\|\sigma\|$ is the number of period cycles of the signature of $\Gamma_{\sigma}$. Given a system of canonical generators of $\Lambda$, let $\left\{c_{i}: i \in I\right\}$ be a set of representatives for the conjugacy classes of reflections in $\Lambda$. 
With these notations, a symmetry $\sigma$ of $X$ with non-empty set of fixed points is conjugate to $\theta\left(c_{j}\right)$ for some $j \in I$ and it was shown in [4] (see also [5]) that it has

$$
\|\sigma\|=\sum\left[\mathrm{C}\left(\theta(\Lambda), \theta\left(c_{i}\right)\right): \theta\left(\mathrm{C}\left(\Lambda, c_{i}\right)\right)\right]
$$

ovals, where the sum is taken over all elements $i$ of $I$ for which $\theta\left(c_{i}\right)$ is conjugate to $\sigma$. The index $w_{i}=w_{i}^{X}=\left[\mathrm{C}\left(\theta(\Lambda), \theta\left(c_{i}\right)\right): \theta\left(\mathrm{C}\left(\Lambda, c_{i}\right)\right)\right]$ will be called a contribution of $c_{i}$ to $\|\sigma\|$.

Now let $\|X\|$ be the sum of all $\|\sigma\|$, where $\sigma$ is running over all conjugacy classes of symmetries of $X$. From (4) it follows immediately that

$$
\|X\|=\sum_{i \in I}\left[\mathrm{C}\left(\theta(\Lambda), \theta\left(c_{i}\right)\right): \theta\left(\mathrm{C}\left(\Lambda, c_{i}\right)\right)\right]
$$

In this context $w_{i}$ will be called a contribution of $c_{i}$ to $\|X\|$ or we shall say simply that $c_{i}$ contributes to $X$ with $w_{i}$ ovals.

Singerman [16] proved that the centralizer $\mathrm{C}\left(\Lambda, c_{j}\right)$ of a canonical reflection $c_{j}$ in an NEC group $\Lambda$ is

$$
\left\langle c_{j}\right\rangle \times\left\langle e_{j}\right\rangle=\mathbb{Z}_{2} \times \mathbb{Z}
$$

if $c_{j}$ corresponds to an empty period cycle and

$$
\left\langle c_{0}\right\rangle \times\left(\left\langle\left(c_{0} c_{1}\right)^{n_{1} / 2}\right\rangle *\left\langle e^{-1}\left(c_{s-1} c_{s}\right)^{n_{s} / 2} e\right\rangle\right)=\mathbb{Z}_{2} \times\left(\mathbb{Z}_{2} * \mathbb{Z}_{2}\right)
$$

or

$$
\left\langle c_{j}\right\rangle \times\left(\left\langle\left(c_{j-1} c_{j}\right)^{n_{j} / 2}\right\rangle *\left\langle\left(c_{j} c_{j+1}\right)^{n_{j+1} / 2}\right\rangle\right)=\mathbb{Z}_{2} \times\left(\mathbb{Z}_{2} * \mathbb{Z}_{2}\right)
$$

if $c_{j}$ corresponds to a period cycle $\left(n_{1}, \ldots, n_{s}\right)$ with even link periods, where $j=0$ or $j \neq 0$ respectively. We are ready to state and prove the main result of the paper.

Theorem 4.1. Let $\sigma_{1}, \ldots, \sigma_{k}$ be non-conjugate symmetries of a Riemann surface $X$ of genus $g \geq 2$ for which $G=$ Aut $^{ \pm}(X)$ is a 2-group. Then $\left\|\sigma_{1}\right\|+\cdots+\left\|\sigma_{k}\right\| \leq 2 g-2+(9-k)|G| / 8$.

Proof. Let $X=\mathcal{H} / \Gamma$ and $G=\Lambda / \Gamma$. Assume that $\Lambda$ has signature of a general form

$$
\left(g^{\prime} ; \pm ;\left[m_{1}, \ldots, m_{r}\right] ;\left\{C_{1}, \ldots, C_{m},(-), . . .,(-)\right\}\right),
$$


where $C_{i}=\left(n_{i 1}, \ldots, n_{i s_{i}}\right)$ and denote $s=s_{1}+\cdots+s_{m}$. Observe that every link period is a power of 2 . Let $\theta: \Lambda \longrightarrow G$ be the canonical epimorphism.

Assume first that none of $\sigma_{1}, \ldots, \sigma_{k}$ is central. Then $\left|\mathrm{C}\left(G, \sigma_{i}\right)\right| \leq$ $|G| / 2$ for $i \leq k$. So any canonical reflection $c$ corresponding to an empty period cycle contributes to $\|X\|$ with at most $|G| / 4$ ovals, by (6) and (5) whilst a reflection corresponding to a non-empty period cycle contribute to $\|X\|$ with at most $|G| / 8$ ovals by (5) and (7) or (8). So $\|X\| \leq(2 l+s)|G| / 8$. On the other hand $g-1 \geq(4 l+4 m-8+s)|G| / 8$ by the Hurwitz-Riemann formula as $\mu(\Lambda) \geq 2 \pi(l+m-2+s / 4)$. Thus since $k \leq l+s$ we obtain $6 l+8 m+s>7+k$ since for $m=0$ we have $l \geq k \geq 9$. Consequently

$$
\begin{aligned}
\|X\| & \leq(2 s+8 l+8 m-16) \frac{|G|}{8}+(16-6 l-8 m-s) \frac{|G|}{8} \\
& \leq 2 g-2+(9-k) \frac{|G|}{8} .
\end{aligned}
$$

So we can assume that some of the symmetries in question, say $z$, is a central element of $G$. Furthermore we can assume that $l=0$ and $m=1$. Observe first that $m \neq 0$. Indeed if $m=0$ then as above we prove that $\|X\| \leq l|G| / 2$ and $2 g-2 \geq|G|(l-2)$. So

$$
\begin{aligned}
\|X\| & \leq l \frac{|G|}{2} \\
& =|G|(l-2)+(4-l) \frac{|G|}{2} \\
& \leq 2 g-2+(16-4 l) \frac{|G|}{8} \\
& <2 g-2+(9-k) \frac{|G|}{8}
\end{aligned}
$$

since $4 l-k>7$ as $l \geq k \geq 9$. Thus we can assume that $m>0$ because otherwise the theorem holds.

We can assume that $\theta\left(c_{10}\right) \neq z$. If $l \neq 0$ consider an NEC group $\Lambda^{\prime}$ with signature

$$
\begin{array}{r}
\left(g^{\prime} ; \pm ;\left[m_{1}, \ldots, m_{r}\right] ;\left\{\left(2,2,2,2, n_{11}, \ldots, n_{1 s_{1}}\right), C_{2}, \ldots, C_{m}\right.\right. \\
(-), !-1,(-)\}) .
\end{array}
$$

For the sake of technical simplicity, we denote in the same way as in the group $\Lambda$ some of the canonical generators of $\Lambda^{\prime}$; namely those generators 
which correspond to "pieces" of the signature of $\Lambda$ in the signature of $\Lambda^{\prime}$ and for the sake of terminological convenience we shall refer to these generators of $\Lambda^{\prime}$ as old generators. To be more precise, this means here in the case of the signatures (9) and (10) that the hyperbolic generators of $\Lambda^{\prime}$ are $a_{1}, b_{1}, \ldots, a_{g^{\prime}}, b_{g^{\prime}}$ or $d_{1}, \ldots, d_{g^{\prime}}$ according to whether the sign is + or - , the elliptic generators are $x_{1}, \ldots, x_{r}$, generators corresponding to the first nonempty period cycle are $e_{1}, c_{0}^{\prime}, c_{1}^{\prime}, c_{2}^{\prime}, c_{3}^{\prime}, c_{10}, c_{11}, \ldots, c_{1 s_{1}}$, the generators corresponding to the remaining nonempty period cycles are $e_{i}, c_{i 0}, c_{i 1}, \ldots, c_{i s_{i}}$, whilst generators corresponding to empty period cycles are $e_{m+1}, c_{m+1}, \ldots, e_{m+l-1}, c_{m+l-1}$. Furthermore according to this convention $c_{0}^{\prime}, c_{1}^{\prime}, c_{2}^{\prime}$ and $c_{3}^{\prime}$, are new generators whilst the remaining are old ones. We shall consider separately two cases
a) $\theta\left(c_{m+l}\right) \neq z$
b) $\theta\left(c_{m+l}\right)=z$.

Case a). Here we define $\theta^{\prime}: \Lambda^{\prime} \longrightarrow G$ on all old canonical generators but $e_{1}$ by $\theta$ and we put $\theta^{\prime}\left(e_{1}\right)=\theta\left(e_{1} \cdots e_{m+l}\right) \theta\left(e_{2} \cdots e_{m+l-1}\right)^{-1}, \theta^{\prime}\left(c_{0}^{\prime}\right)=$ $\theta^{\prime}\left(e_{1}^{-1} c_{1 s_{1}} e_{1}\right), \theta^{\prime}\left(c_{1}^{\prime}\right)=\theta^{\prime}\left(c_{3}^{\prime}\right)=z$, and $\theta^{\prime}\left(c_{2}^{\prime}\right)=\theta\left(c_{m+l}\right)$. Then, using results of [3, Chapter 2], it is not difficult to see that $\Gamma^{\prime}=\operatorname{Ker} \theta^{\prime}$ is a Fuchsian surface group. Indeed, by Theorem 2.2.4, its signature has no proper periods, by Theorem 2.3.3, it has no link periods, and finally, by Theorem 2.1.3, its sign is + . Let $X^{\prime}=\mathcal{H} / \Gamma^{\prime}$. As $\mu(\Lambda)=\mu\left(\Lambda^{\prime}\right)$ we see that $X$ and $X^{\prime}$ have the same genus. We shall show that $\left\|X^{\prime}\right\| \geq\|X\|$.

As the images under $\theta^{\prime}$ of all old, except $c_{10}$, canonical reflections corresponding to nonempty period cycles and their neighbours are the same as their images under $\theta$ we see, by (5) and (7) or (8), that each of these reflections contributes to $X^{\prime}$ with the same number of ovals as to $X$. Similarly, by (6) and (5), old reflections corresponding to empty period cycles contribute to $X^{\prime}$ with the same number of ovals as to $X$. So we have to show that $c_{10}, c_{0}^{\prime}, c_{1}^{\prime}, c_{2}^{\prime}$ and $c_{3}^{\prime}$ contribute all together to $X^{\prime}$ with at least as many ovals as $c_{m+l}$ and $c_{10}$ contribute to $X$.

Let $w_{10}$ be the contribution of $c_{10}$ to $\|X\|$. Then $c_{10}$ contributes to $X^{\prime}$ with $w_{10}$ or $w_{10} / 2$ ovals according to whether $\theta\left(c_{10} c_{11}\right)^{n_{11} / 2}=z$ or not. Similarly $c_{0}^{\prime}$ contributes to $X^{\prime}$ with $w_{10}$ or $w_{10} / 2$ ovals according to whether $\theta\left(c_{1 s_{1}-1} c_{1 s_{1}}\right)^{n_{1 s_{1}} / 2}=z$ or not. Consequently reflections $c_{10}$ and $c_{0}^{\prime}$ contribute to $\theta^{\prime}\left(c_{10}\right)$ at least the same number of ovals as $c_{10}$ to $\theta\left(c_{10}\right)$.

Assume now, that $c_{m+l}$ had contributed with $k$ ovals to $\theta\left(c_{m+l}\right)$. Then $c_{2}^{\prime}$ contributes to the new surface $X^{\prime}$ also with $k$ ovals if $\theta\left(e_{m+l}\right) \neq$ 1 and in this case we are done since the new surface has at least the same number of ovals as the former one. If $\theta\left(e_{m+l}\right)=1$ then $c_{2}^{\prime}$ contribute to $X^{\prime}$ with $k / 2$ ovals. Let $n^{\prime}$ and $n^{\prime \prime}$ be the orders of $\theta^{\prime}\left(c_{0}^{\prime}\right) \theta^{\prime}\left(c_{2}^{\prime}\right)$ 
and $\theta^{\prime}\left(c_{2}^{\prime}\right) \theta^{\prime}\left(c_{10}\right)$ respectively and let $n=\max \left\{n^{\prime}, n^{\prime \prime}\right\}$. Then the centralizer of $\theta\left(c_{m+l}\right)$ had order not bigger than $2|G| / n$ by the Lemma 3.2 and so $c_{m+l}$ had contributed to the former surface at most with $|G| / n$ ovals, i.e., $k \leq|G| / n$ whilst now $c_{1}^{\prime}$ and $c_{3}^{\prime}$ contribute to $z$ with $|G| / 4 n^{\prime}+|G| / 4 n^{\prime \prime} \geq|G| / 2 n \geq k / 2$ ovals on the new surface $X^{\prime}$. So indeed $\left\|X^{\prime}\right\| \geq\|X\|$.

Case b). If $\theta\left(c_{m+l}\right)=z$ then we define $\theta^{\prime}: \Lambda^{\prime} \longrightarrow G$ on all old canonical generators and on $c_{0}^{\prime}$ as for the case $\theta\left(c_{m+l}\right) \neq z$ and we put $\theta^{\prime}\left(c_{1}^{\prime}\right)=\theta^{\prime}\left(c_{3}^{\prime}\right)=\theta\left(c_{m+l}\right)$, and $\theta^{\prime}\left(c_{2}^{\prime}\right)=\theta\left(c_{10}\right)$. Again, using results of [3, Chapter 2] one can prove that $\Gamma^{\prime}=\operatorname{Ker} \theta^{\prime}$ is a Fuchsian surface group and by the Hurwitz-Riemann formula $X^{\prime}=\mathcal{H} / \Gamma^{\prime}$ is a Riemann surface of genus $g$. We shall show that $\left\|X^{\prime}\right\| \geq\|X\|$. Also here all old canonical reflections but $c_{10}$ contribute to $X^{\prime}$ with the same number of ovals as to $X$. The new reflection $c_{2}^{\prime}$ contributes to $X^{\prime}$ with no less ovals than $c_{10}$ to $X$. Here $c_{m+l}$ had contributed to $\theta\left(c_{m+l}\right)$ with $|G| / 4$ or $|G| / 2$ ovals according as $\theta\left(e_{m+l}\right) \neq 1$ or $\theta\left(e_{m+l}\right)=1$. In the first case we see that $\left\|X^{\prime}\right\| \geq\|X\|$ as $c_{3}^{\prime}$ contribute to $X^{\prime}$ with $|G| / 4$ ovals also. If $\theta\left(e_{m+l}\right)=1$, then $\theta^{\prime}\left(e_{1}\right)=\theta\left(e_{1}\right)$. So in this case $\theta^{\prime}\left(c_{0}^{\prime}\right)=\theta\left(c_{10}\right)$ and therefore $c_{1}^{\prime}$ and $c_{3}^{\prime}$ contribute to $X^{\prime}$ with $|G| / 4$ ovals each. Hence again $\left\|X^{\prime}\right\| \geq\|X\|$.

Thus we can assume that $\Lambda$ has no empty period cycles, i.e., it has signature

$$
\left(g^{\prime} ; \pm ;\left[m_{1}, \ldots, m_{r}\right] ;\left\{\left(n_{11}, \ldots, n_{1 s_{1}}\right), \ldots,\left(n_{m 1}, \ldots, n_{m s_{m}}\right)\right\}\right) .
$$

Now we shall see that, actually we can assume that $m=1$, i.e., $\Lambda$ has just one period cycle. For, observe that we can assume that $\theta\left(c_{1 s_{1}}\right) \neq z$ and $\theta\left(c_{20}\right) \neq z$. Let $\Lambda^{\prime}$ be an NEC group with signature

$$
\begin{array}{r}
\left(g^{\prime} ; \pm ;\left[m_{1}, \ldots, m_{r}\right] ;\left\{\left(n_{11}, \ldots, n_{1 s_{1}}, 2,2, n_{21}, \ldots, n_{2 s_{2}}, 2,2\right)\right.\right. \\
\left.\left.C_{3}, \ldots, C_{s}\right\}\right) .
\end{array}
$$

Here the reflections corresponding to the first period cycle are

$$
c_{10}, \ldots, c_{1 s_{1}}, c_{0}^{\prime}, c_{20}, \ldots, c_{2 s_{2}}, c_{1}^{\prime}, c_{2}^{\prime}
$$

and also here $\mu(\Lambda)=\mu\left(\Lambda^{\prime}\right)$. We define $\theta^{\prime}: \Lambda^{\prime} \longrightarrow G$ on all old canonical generators but $e_{1}$ as before i.e., by $\theta$ and we put $\theta^{\prime}\left(e_{1}\right)=$ $\theta\left(e_{1}\right) \theta\left(e_{2}\right)$. Furthermore we define $\theta^{\prime}\left(c_{0}^{\prime}\right)=\theta^{\prime}\left(c_{1}^{\prime}\right)=z$ and $\theta^{\prime}\left(c_{2}^{\prime}\right)=$ $\theta^{\prime}\left(e_{1}\right) \theta\left(c_{10}\right) \theta^{\prime}\left(e_{1}^{-1}\right)$. Once more, using results of [3, Chapter 2], we 
see that $\Gamma^{\prime}=\operatorname{Ker} \theta^{\prime}$ is a Fuchsian surface group. Then $X^{\prime}=\mathcal{H} / \Gamma^{\prime}$ is a Riemann surface of genus $g$. In a similar way, we can prove that $\left\|X^{\prime}\right\| \geq\|X\|$. Indeed all old canonical reflections, but $c_{10}$ and $c_{20}$ contribute to $X^{\prime}$ with the same number of ovals as to $X$.

Let $w_{i}^{X}$ be the contribution of $c_{i 0}$ to $\|X\|$ and let $l_{i}$ be the order of the centralizer of $\theta\left(c_{i 0}\right)$ for $i=1,2$. Then $w_{i}^{X}=l_{i} / 4 k_{i}$, where $k_{i}$ is the order of $\theta\left(c_{i 0} c_{i 1}\right)^{n_{i 1} / 2} \theta\left(e_{i}^{-1}\left(c_{i s_{i}-1} c_{i s_{i}}\right)^{n_{i s_{i}} / 2} e_{i}\right)$. In particular we see that $w_{i}^{X} \leq l_{i} / 4$. On the other hand, as $\left.\theta^{\prime}\left(c_{10} c_{11}\right)\right)^{n_{11} / 2} \theta^{\prime}\left(e_{1}^{-1} c_{1}^{\prime} c_{2}^{\prime} e_{1}\right)$ and $\theta^{\prime}\left(c_{1 s_{1}-1} c_{1 s_{1}}\right)^{n_{1 s_{1}} / 2} \theta^{\prime}\left(c_{1 s_{1}} c_{0}^{\prime}\right)$ have order 2 we see that $c_{10}$ and $c_{1 s_{1}}$ contribute to $X^{\prime}$ with no less ovals than $c_{10}$ to $X$. Similarly $c_{20}$ and $c_{2 s_{2}}$ contribute to $X^{\prime}$ with no less ovals than $c_{20}$ to $X$. So we see that indeed $\left\|X^{\prime}\right\| \geq\|X\|$.

So at last we arrive at the case of an NEC group $\Lambda$ with signature

$$
\left(g^{\prime} ; \pm ;\left[m_{1}, \ldots, m_{r}\right] ;\left\{\left(n_{1}, \ldots, n_{s}\right)\right\}\right) \text {. }
$$

Let $c_{0}, \ldots, c_{s}$ denote the corresponding canonical reflections. Observe that $s \leq 8(g-1) /|G|+4$.

We can assume that $\theta\left(c_{0}\right)$ is a central symmetry of $X$ and so in particular $\theta\left(c_{0}\right)=\theta\left(c_{s}\right)$. Consider $c_{0}, c_{1}, \ldots, c_{s-1}$ as $s$ points on a circle labelled by $\theta\left(c_{0}\right), \theta\left(c_{1}\right), \ldots, \theta\left(c_{s-1}\right)$ respectively. By the Lemma 3.3 , at least for $k-1$ numbers in range $0 \leq i_{1}<\cdots<i_{k-1} \leq s-1$, $\theta\left(c_{i_{t}-1}\right) \neq \theta\left(c_{i_{t}+1}\right)$, where the indices are taken modulo $s$.

Now if $n_{i_{t}}>2$ or $n_{i_{t}+1}>2$ then $\theta\left(c_{i_{t}}\right)$ is not central and so $\left|\mathrm{C}\left(G, \theta\left(c_{i_{t}}\right)\right)\right| \leq|G| / 2$. Therefore $c_{i_{t}}$ contributes to the corresponding surface $X$ with at most with $|G| / 8$ ovals. If $n_{i_{t}}=n_{i_{t}+1}=2$ then $\left|\theta\left(\mathrm{C}\left(\Lambda, c_{i_{t}}\right)\right)\right| \geq 8$ and thus also now $c_{i_{t}}$ contributes to $X$ with at most $|G| / 8$ ovals. The remaining canonical reflections contribute to $X$ with no more than $|G| / 4$ ovals. So

$$
\begin{aligned}
\|X\| & \leq(k-1) \frac{|G|}{8}+(s-k+1) \frac{|G|}{4} \\
& =s \frac{|G|}{4}+(1-k) \frac{|G|}{8} \\
& \leq 2 g-2+|G|+(1-k) \frac{|G|}{8} \\
& =2 g-2+(9-k) \frac{|G|}{8} .
\end{aligned}
$$

This completes the proof. 
Corollary 4.2. Let $\sigma_{1}, \ldots, \sigma_{k}$, where $k \geq 9$ be non-conjugate symmetries of a Riemann surface $X$ of genus $g \geq 2$. Then $\left\|\sigma_{1}\right\|+\cdots+\left\|\sigma_{k}\right\| \leq$ $2 g-2+2^{r-3}(9-k)$, where $r$ is the smallest positive integer for which $k \leq 2^{r-1}$.

Proof. As we are looking for the ovals of these symmetries and conjugate symmetries have the same number of ovals we can assume, using Sylow theorem, that they generate a 2-subgroup $G$ of $\operatorname{Aut}^{ \pm}(X)$. Let $X=\mathcal{H} / \Gamma$ and $G=\Lambda / \Gamma$. Assume that $\Lambda$ has signature (9). Then, as $s+l \geq k \geq 9$, we see, by [2] (see also [3, Theorem 2.4.7]), that its signature is maximal. So by [3, Theorem 5.1.2] there exists a maximal NEC group $\Lambda^{\prime}$ and algebraic isomorphism $\varphi: \Lambda \longrightarrow \Lambda^{\prime}$. Let $X^{\prime}=\mathcal{H} / \Gamma^{\prime}$, where $\Gamma^{\prime}=\varphi(\Gamma)$. Then $\operatorname{Aut}^{ \pm}\left(X^{\prime}\right)=\Lambda^{\prime} / \Gamma^{\prime}$ and $\varphi$ induces an isomorphism $\tilde{\varphi}: \Lambda / \Gamma \longrightarrow \Lambda^{\prime} / \Gamma^{\prime}$. Now $\widetilde{\varphi}\left(\sigma_{1}\right), \ldots, \widetilde{\varphi}\left(\sigma_{k}\right)$ are nonconjugate symmetries of $X^{\prime}$. Furthermore if $\left\langle\sigma_{i}\right\rangle=\Lambda_{i} / \Gamma$, then $\left\|\sigma_{i}\right\|$ is the number of empty period cycles of $\Lambda_{i}$. So $\left\|\sigma_{i}\right\|=\left\|\widetilde{\varphi}\left(\sigma_{i}\right)\right\|$ since $\left\langle\widetilde{\varphi}\left(\sigma_{i}\right)\right\rangle=\varphi\left(\Lambda_{i}\right) / \Gamma^{\prime}$. Furthermore $\|X\| \leq\left\|X^{\prime}\right\|$ and $G \cong \operatorname{Aut}^{ \pm}\left(X^{\prime}\right)$ is a 2-group. Then by Theorem 4.1, $\left\|X^{\prime}\right\| \leq 2 g-2+(9-k)|G| / 8$ and by Lemma 3.1, $|G| \geq 2^{r}$. Hence the Corollary follows.

The next theorem shows that the bound obtained in Corollary 4.2 is sharp.

Theorem 4.3. Let $k \geq 9$ be an arbitrary integer and let $r$ be the smallest positive integer for which $k \leq 2^{r-1}$. Then for arbitrary $g=$ $2^{r-2} t+1$, where $t \geq k-3$ there exists a Riemann surface $X$ of genus $g$ having $k$ non-conjugate symmetries which have $2 g-2+2^{r-3}(9-k)$ ovals in total.

Proof. Let $G=\mathbb{Z}_{2} \oplus \stackrel{r}{ } \cdot \mathbb{Z}_{2}=\left\langle z_{1}\right\rangle \oplus \cdots \oplus\left\langle z_{r}\right\rangle$ and let $\Lambda$ be a maximal NEC-group with signature $(0 ;+;[-] ;\{(2, .2 s .2)\})$, where $s=$ $(g-1) / 2^{r-2}+2 \geq k-1$. Let $\left\{a_{1}, \ldots, a_{2^{r-1}}\right\}$ be all elements of order 2 in $G$ which have odd length in $z_{1}, \ldots, z_{r}$ and assume that $a_{1}, \ldots, a_{r}$ generate $G$. Then since $r$ is the minimal integer such that $k \leq 2^{r-1}$ we have $k \geq r$ and so the assignment

$$
\theta(e)=1, \text { and } \theta\left(c_{i}\right)= \begin{cases}a_{1}, & \text { for } i=2 j, 0 \leq j \leq s, \\ a_{j+2}, & \text { for } i=2 j+1,0 \leq j \leq k-2, \\ a_{k}, & \text { for } i=2 j+1, k-1 \leq j \leq s-1,\end{cases}
$$


defines an epimorphism $\theta: \Lambda \longrightarrow G$ for which $\Gamma=\operatorname{Ker} \theta$ is a surface group and $X=\mathcal{H} / \Gamma$ is a Riemann surface having $k$ non-conjugate symmetries with fixed points.

We see that $c_{2 j}$, for $0 \leq j \leq k-2$ contribute to $a_{1}$ with $2^{r-3}$ ovals whilst the remaining $2 s-k+1$ non-conjugate canonical reflections of $\Lambda$ contribute to the corresponding surface with $2^{r-2}$ ovals. As a result

$$
\begin{aligned}
\left\|\sigma_{1}\right\|+\cdots+\left\|\sigma_{k}\right\| & =2^{r-3}(k-1)+2^{r-2}(2 s-k+1) \\
& =2^{r-1} s+2^{r-3}(1-k) \\
& =2 g-2+2^{r}+2^{r-3}(1-k) \\
& =2 g-2+2^{r-3}(9-k) .
\end{aligned}
$$

Acknowledgements. The author is gratefull to the referees for their comments and suggestions.

\section{References.}

[1] Alling, N. L., Greenleaf, N., Foundations of the Theory of Klein Surfaces. Lecture Notes in Math. 219, Springer-Verlag, 1971.

[2] Bujalance, E., Normal NEC signatures. Illinois J. Math. 26 (1982), 519-530.

[3] Bujalance, E., Etayo, J. J., Gamboa, J. M., Gromadzki, G., Automorphisms Groups of Compact Bordered Klein Surfaces, A Combinatorial Approach. Lecture Notes in Math. 1439, Springer-Verlag, 1990.

[4] Gromadzki, G., Groups of Automorphisms of Compact Riemann and Klein Surfaces. University Press WSP Bydgoszcz, 1993.

[5] Gromadzki, G., On a Harnack-Natanzon theorem for the family of real forms of Rieamnn surfaces. J. Pure Appl. Alg. 121 (1997), 253-269.

[6] Gromadzki, G., Izquierdo, M., Real forms of a Riemann surface of even genus. Proc. Amer. Math. Soc. 126 (1998), 3475-3479.

[7] Gromadzki, G., Izquierdo, M., On ovals of Riemann surfaces of even genera. Geometriae Dedicata 78 (1999), 81-88.

[8] Harnack, A., Über die Vieltheiligkeit der ebenen algebraischen Kurven. Math. Ann. 10 (1876), 189-199.

[9] Macbeath, A. M., The classification of non-euclidean crystallographic groups. Can. J. Math. 19 (1967), 1192-1205.

[10] May, C. L., Automorphisms of compact Klein surfaces with boundary. Pacific J. Math. 59 (1975), 199-210. 
[11] Natanzon, S. M., Automorphisms of the Riemann surface of an $M$-curve. Funktsional Anal. i Priloz. 12 (1978), 82-83. Functional Anal. Appl. 12 (1978), 228-229.

[12] Natanzon, S. M., On the total number of ovals of real forms of complex algebraic curves. Uspekhi Mat. Nauk 35 (1980), 207-208. Russian Math. Surveys 35 (1980), 223-224.

[13] Natanzon, S. M., Finite groups of homeomorphisms of surfaces and real forms of complex algebraic curves. Trudy Moscov Mat. Obsch 51 (1988), 3-53. Trans. Moscow Math. Soc. 51 (1989), 1-51.

[14] Natanzon, S. M., Harnack type theorem for families complexely isomorphic real algebraic curves. Uspekhi Mat. Nauk 52 (1997), 173-174. Russian Math. Surveys 52 (1997), 1314-1315.

[15] Preston, R., Projective structures and fundamental domains on compact Klein surfaces. Ph. D. Thesis. University of Texas, 1975.

[16] Singerman, D., On the structure of non-euclidean crystallographic groups. Proc. Camb. Phil. Soc. 76 (1974), 233-240.

[17] Singerman, D., Mirrors on Riemann surfaces. Contemporary Mathematics 184 (1995), 411-417.

[18] Wilkie, H. C., On non-euclidean crystallographic groups. Math. Z. 91 (1966), 87-102.

Recibido: 5 de febrero de 1.999

\author{
Grzegorz Gromadzki* \\ Institute of Mathematics \\ University of Gdańsk \\ Wita Stwosza 57 \\ 80-952 Gdańsk, POLAND \\ greggrom@ksinet.univ.gda.pl
}

\footnotetext{
* Supported by the DGICYT through the grant Año sabatico 97/98
} 